\title{
Study on Increasing Mental Capital of Left-behind Children in Rural Areas - A Case Study of Caring for Children Left Behind in "Aijia Social Studio"
}

\author{
Fuyu Du
}

Xinyu University, Xinyu, Jiangxi, 338004

Keywords: Metal Capital, Left-behind Children, Rural Areas

\begin{abstract}
Based on the social work project of Caring for Left-behind Children supported by Central Government, the research selects a group of left-behind children in Hutou Primary School, Yushu of Xinyu City as the object, and carries out a series of group exploration activities to enhance the psychological capital left behind children, Children's hearts are taken care of, to meet the spiritual needs of left-behind children and the needs of the community, enhance the ability to adapt to life, increasing the psychological capital to deal with life.
\end{abstract}

\section{Introduction}

Left-behind children in rural areas refer to children under the age of 16, migrant workers on one or both of their parents, children left in their hometown, and not living with both parents. That is, they did not follow their parents in the place where they were employed and received education. Instead, they were left in the local rural areas and were children of rural migrant workers who went to school. As parents go out to work, the distance between rural left-behind children and their parents increases, and the stretches of time stretch away. It is hard to "enjoy" the happiness that ordinary children can easily obtain. The absence of parents will affect the cultivation of children's sound personality. The absence of parents easily causes children's self-esteem and loneliness to increase. They are reluctant to contact with the outside world and their parents' care and affection are lacking and their self-esteem is affected. Aiming at the psychological problems that left-behind children are prone to appear, this study, based on the social work project of Love Family Social Office, applied to the left behind children supported by the Central Government, selected the left-behind children in Hutou Primary School of Yushu New District as the targets, Psychological capital action research. In order to make the action research pertinence, the research first investigated the psychological situation of the left-behind children in the lake primary school, learned the situation through the left-behind children's teachers and guardians, and then conducted a series of action research.

\section{Pre-investigation found that the main psychological problems of children left behind}

Inferior psychological more common. According to school teachers, the vast majority of children left behind in rural areas have a more serious degree of inferiority complex. Because of their long-term presence, left-behind children feel less rely on and strong protection around, see the parents are accompanied by children, prone to inferiority. Psychological long-term backlog of feelings of inferiority will have more negative emotions experience, not living with their parents, often feel very lonely, prone to depression in the heart of unhealthy psychology, which for the child's future study, life and physical and mental health are Very unfavorable.

Heart more closed, interpersonal relationships affected. Left-behind children are often unable to conduct psychological exchanges with their parents due to their long-term absence. They lack the opportunity to talk and seek help. Their inner worries and conflicts are incapable of being talked and can not be guided. They can not form a sense of security and trust in the outside world, for a long time, it is easy to become timid, cowardly, closed-hearted, apathetic, withdrawn and lack of initiative in communication, thus seriously affecting the quality of their social interaction with others. Children in their daily lives because there is no parental constraints, more prone to 
misconduct, manifested as irritable, impulsive and irritable, and often no problem escalated to.

Serious reverse psychology. Left-behind children lack a sense of security in the heart, psychological more sensitive to the relationship between people is full of distrust, more likely to produce rebellious, obvious emotional confrontation. This kind of subconscious mistrust affects children's judgment of the interpersonal environment, and sometimes they feel abandoned and feel psychologically negative. In the face of the unwillingness of day-to-day teaching of family members and teachers to act in concert, they often collide with teachers and parents to vent their resistance. Many left-behind children behaved poorly in school. There were more and more disciplinary phenomena. They often became less and less cohesive, their collectivism was relatively weak, and some even developed bad habits such as smoking, drinking and indulging in online games. Their emotions fluctuate widely, their attitudes are extremely unbalanced, and they are easily injured in social interactions with others.

The custodians of left-behind children are generally the elderly or unilaterally supervised by their mothers. If both parents are migrant workers, the elderly generally can only take care of their children's lives and can not learn and mentally guide them. Even mothers who are at home may find it harder to supervise the higher grades Therefore, left behind children generally lack of motivation to learn, interest in learning is not strong, aggressive, disciplined, coupled with no counseling at home, generally poor academic performance. Parents can not often communicate around, guiding their own direction, more confused heart, a great waste of time, can not be effectively used to learning.

\section{To enhance the psychological capacity of left-behind children's action measures}

The researchers participated in the "Growing with You" project of caring for left-behind children. The project selected left-behind children in Yushuu Primary School of Xinyu City as the target. The purpose of the action focused on how "schools, parents and social organizations" Children accompany, respect, listen, accept, concern, thankful!

Social workers who recruit various professional backgrounds and skills include teachers, counselors, lawyers and community workers. Every week to Xinyu City, Lake primary school psychological quality training activities.

As of November 2017, Aijia Social Studio has conducted over a dozen special team activities. Before the event, the project team carefully prepared and designed a program for each activity, covering various topics such as the theme of the activity, the background of the activity, the content of the activity, the purpose of the activity, and the preparation for the activities. Now cite one of the planning programs: "to enhance the exploration of psychological capital" with your growth, "one by one honed toughness chapter."

Activity theme: Lake primary care "children left behind" and "one to one help." Background: Nowadays, the society is more and more demanding of group collaboration. Our children should foster the spirit of group collaboration and lay a good foundation for their future access to the community. Group collaboration requires that children exercise and develop in group activities. Through the participation of team activities, so that children understand how to arrange, how the division of labor, how to collaborate. Through the game makes the child has a profound insight. Activities: Positive Psychology Group activities - to build the tower. Purpose of the activity: Through children's division of labor, let the children know when to accept a task need to be arranged. And through the children did not do each other to complete the tower construction, experience in the process of building the tower how to adjust, how to cooperate and how to organize everyone involved, let children understand the importance of group collaboration.

Activities. Special activities to enhance psychological capital are: self-confidence articles, toughness articles, team cohesion articles, optimistic articles, hope articles, strong articles, anti-stress articles, social skills articles, articles of responsibility, self motivation articles, special activities, sometimes Will be appropriate to repeat, to enhance the psychological quality of the previous activity training effect. Organization of the organization activities or games activities can keep children's minds grow, such as "Pearl River Wanli" through the common transmission of a ball, 
so that we feel effective teamwork, Cohesion and self-control ability, understand life, learning, for a common purpose and the sense of responsibility of the team, everyone should make their own part in order to make this team become strong; show of hands, ask each person's two Only arms straight to the chest flat, the body is not allowed to shake, insist for a few minutes, to see who can persist to the end, which left behind children to experience the patience and perseverance needed to persevere, develop their willpower, cultivate left-behind children unconvinced , Not to be outdone, inspire them to overcome the difficulties in their studies and life.

Activity process. Every time when going to Huguang Primary School to promote the psychological capital exploration group for left-behind children, warm-up activities should generally be carried out to harmonize the atmosphere and narrow the psychological distance between volunteers and children in order to carry out exploration group activities smoothly. Followed by each special group to explore activities, to carry out related group activities, such as self-confidence optimistic articles "looking for" people "," do not pour the forest ", honed tough articles - to build tower, team cohesion articles - psychological development game -" beads Miles ", optimistic articles -" Giant feet. " After the group activity is finished, the children carry out the sharing activity experience, summarize the activities and gain the spiritual growth and increase the psychological capital of the others.

Activity form. Group activities have selected a grade two classes a class two classes, sometimes divided according to the needs of group activities, sub-group. Group activities sometimes involve guardians of left-behind children to participate, so that guardians can better understand children's psychological needs and increase their knowledge of their children. In order to let left-behind children get personalized growth, often after the group activities to carry out one-on-one counseling, individual counseling are generally in the early activities, observed some left-behind children lack the positive side of the sun, and then targeted counseling. Family visits are also sometimes carried out, going deep into children's homes and learning about children's life.

\section{Activities and gains}

Through the project team to accompany their children's soul for nearly a year, the left-behind children in the Lake Primary School become sunshine, positive and progressive. They are optimistic about learning and living. Their expressions are obviously more confident and open-minded to their classmates and elders. Learning interest increased, the heart is not confused.

Stay with children learning life, the soul get care. The separation of parents led to the incomplete family structure of left-behind children. Most of their guardianship and guardianship were ancestors. They could only meet the material needs of the left-behind children and the lack of parenting education, which affected the normal psychological growth of left-behind children in varying degrees. Nowadays, a group of volunteers with love, professional work and professional help skills are regularly accompanying children left behind to bring joy, warmth and sunshine to the left-behind children. In the activities, all kinds of negative emotions accumulated in the ordinary days are effectively diverted, Bad behavior was corrected, cultivating a positive psychological left-behind children.

To meet the spiritual needs of left-behind children and social needs. The absence of parents makes it hard for rural left-behind children to obtain basic affection. After generations of support, left-behind children in rural areas can not form lifestyles and values that are compatible with them. Parent-child alienation can create an indelible shadow on left-behind children in rural areas and lonely. Now there is such a group of caring people to accompany them to learn and live, to fill the spiritual emptiness and soothe the injured mentality. Through a series of activities, left-behind children feel interpersonal care, get careful care, improve the ability to adapt to life, personality has been improved.

To enhance the ability to adapt to all kinds of life, increasing the psychological capital to deal with life. Hutou Primary Left-behind Children Volunteer Project team plan a series of activities program, regular activities to the primary lake activities, activities in addition to guiding the children left behind to learn, life on the proper material help to God, each action has a special 
psychological quality Enhance training activities, such as overcoming inferiority and self-confidence; teamwork and communication skills training; tempering will, resilience formation; self-motivation, upward spirit of access and so on. For example, through persistent training in "show of hands", we can let children know that we must have strong willpower both in life and in study. We must have the patience and perseverance to do any difficult things before we can overcome difficulties. Do things better. In the "I am really good" "beads line" "fishing" psychological group counseling activities, so that left-behind children learn to dare to express themselves, self-esteem, grow into a confident person. It is through a series of activities to cultivate left-behind children to deal with the psychological capital of life, enhance the ability to adapt to life.

\section{Research reflection}

Service content is limited. "Staying with You" The project of caring for left-behind children mainly focuses on improving the psychological capital of left-behind children. A series of activities planned and carried out by the project are all around this aspect. However, left-behind children need more than psychological quality but also education guidance, Safety and health concerns, social resources and more.

Service time short-term. "With your growth," care for children left behind the project into the closing phase. Although every activity basically achieved the desired effect, the psychological quality of left-behind children in Hutou Primary School did indeed improve. However, with the completion of the project and the completion of the project, there was no continuing activity in the future and the psychological capital harvested could not be maintained. We all know that to form a psychological quality requires long-term efforts, so we must strive to ensure the sustainability of the project.

Unity of service. Projects are basically carried out a number of games and group counseling activities, these single service, help to children left behind is limited. Can be interested in teaching, family services, network communication, case accurate guidance and other means of service.

\section{References}

[1] Ren Maoxian. Study on the Social Work Involved in the Education of Left-behind Children in Rural Areas - A Case Study of a Town in Shandong Province [M]. Liaoning University, 2015.5

[2] He Lei. Research on social work involved in left-behind children in rural areas from the perspective of superiority [M]. Anhui University, 2015.4

[3] Cheng Meiling. Study on the service mode of social work for left-behind children in rural areas A case study of the "warm mountainous area" in village [M]. Anhui University, 2017.3 